\title{
The UV Upturn: From M32 to Distant Clusters
}

Thomas M. Brown (tbrownestsci.edu)

Space Telescope Science Institute, 3700 San Martin Drive, Baltimore, MD 21218, USA

\begin{abstract}
I review the observational constraints on the stars responsible for the upturn in the UV spectra of ellipticals, ranging from galaxies in the local Universe to distant clusters. In nearby galaxies, this UV upturn is produced by a minority population of extreme horizontal branch (EHB) stars, with the large variations observed in the UV-to-optical flux ratio driven by variations in the number of EHB stars, and not the type of UV-bright stars. Deep UV images of the nearest elliptical galaxy, M32, show that it has a well-populated EHB, even though it has the weakest UV upturn of any known elliptical galaxy. However, M32 suffers from a striking dearth of the hot post-HB stars expected from canonical evolutionary theory. As we observe to larger lookback times in more distant galaxy clusters, the UV upturn fades, as predicted by theories of stellar and galactic evolution, but does so gradually. Because the EHB stars do not appear suddenly in the Universe, their presence is likely driven by a large dispersion in the parameters that govern HB morphology.
\end{abstract}

\section{Introduction}

The source of UV emission from elliptical galaxies was one of the great mysteries of extragalactic astrophysics for nearly 30 years. The "UV upturn" manifests itself as a rising flux shortward of $2500 \AA$. Although the UV upturn is usually associated with the spectra of elliptical galaxies, it was actually discovered in the bulge of the nearby spiral M31 (Code, 1969). Prior to those observations, spiral bulges and elliptical galaxies were thought to contain only cool, passively-evolving populations of old stars. Although elliptical galaxies have very similar optical spectra, their UV-to-optical flux ratios, as measured by the $m_{1550}-V$ color index, show strong variations, correlated with metallicity (Burstein et al., 1988), such that galaxies with higher metallicity (optical $\mathrm{Mg}_{2}$ index) are bluer. By 1990, there were many candidates for the source of the UV emission, including young massive stars, binaries, hot white dwarfs, extreme horizontal branch (EHB) stars, post-asymptotic giant branch (post-AGB) stars, and non-thermal activity (Greggio \& Renzini, 1990). Arguments based upon the fuel consumption during different evolutionary phases made EHB stars a likely source, one that implied a strong decline in the UV upturn at increasing redshift ( $z$ ) (Greggio \& Renzini, 1990).

In the past decade, UV observations of elliptical galaxies in both the local and distant Universe have proved conclusively that EHB stars are the source of the UV upturn, and mapped its evolution over the range $0 \leq z \leq 0.6$. In these proceedings, I review this observational evidence. Section 2 reviews the spectroscopic observations of nearby elliptical galaxies, which are wellmatched by the integrated light of EHB stars and their descendents. Section 3

(C) 2018 Kluwer Academic Publishers. Printed in the Netherlands. 
covers the UV imaging of the nearest elliptical galaxy, M32, which resolves the EHB population responsible for the UV upturn but shows a surprising scarcity of the UV-bright stars expected in the later stages of stellar evolution. Section 4 discusses the evolution of the UV upturn with redshift.

\section{Spectroscopy of Nearby Elliptical Galaxies}

During the Astro-1 and Astro-2 missions, the Hopkins Ultraviolet Telescope (HUT) observed six quiescent elliptical galaxies spanning a wide range in $m_{1550}-V$ (Ferguson et al., 1991; Brown et al., 1995; Brown et al., 1997). The fast focal ratio, large apertures, and wavelength coverage down to the Lyman limit made HUT an ideal instrument for observing extended objects and for determining the effective temperatures of hot UV sources. The HUT spectra (figure 1) were inconsistent with young massive stars, having a lack of strong CIV $\lambda \lambda 1548,1551$ absorption and a declining continuum from $1050 \AA$ to the Lyman limit (i.e., implying temperatures less than $25,000 \mathrm{~K}$ for the UV sources). Instead, the spectra were well-matched by the integrated light expected from populations of EHB stars and their descendents.

Although the galaxies in figure 1 span nearly 2 mag in $m_{1550}-V$, their spectra are very similar, and well fit by models with a narrow range in envelope mass on the EHB $\left(0.02-0.09 M_{\odot}\right)$. Neither post-AGB stars (descendents of red HB stars) or post-early AGB stars (descendents of blue HB stars) can contribute significantly to any of these spectra, because their spectra are respectively hotter and cooler than those observed. This demonstrates that the strong variations in the UV emission, relative to the optical, are the result of variations in the fraction of EHB stars in the population, and not a variation in the type of stars producing the UV flux. Moreover, the HB distribution in each galaxy must be strongly bimodal, with a significant but minority $(\lesssim 10 \%)$ population of EHB stars, very few blue HB stars, and a majority population of red HB stars.

The successor to HUT, the Far Ultraviolet Spectroscopic Explorer (FUSE), has been operating since 1999. Although it observed the giant elliptical galaxy NGC1399 (in Fornax) early in the mission (Brown et al., 2002), reaction wheel failures have rendered the Virgo cluster, where nearly all nearby elliptical galaxies reside, virtually unobservable. Thus, observations of M60 were never completed, and observations of additional ellipticals are unlikely. Figure 2 shows the NGC1399 spectrum and the same best-fit EHB model that matched the HUT spectrum, but at higher resolution. Although the resolution for FUSE $(0.025 \AA)$ is much higher than that of HUT $(3 \AA)$, the velocity dispersion in NGC1399 effectively limits the resolution to $\sim 1 \AA$. Nevertheless, the increase in resolution and signal-to-noise allows a determination of the photospheric abundances for the EHB stars driving the UV upturn. The C 


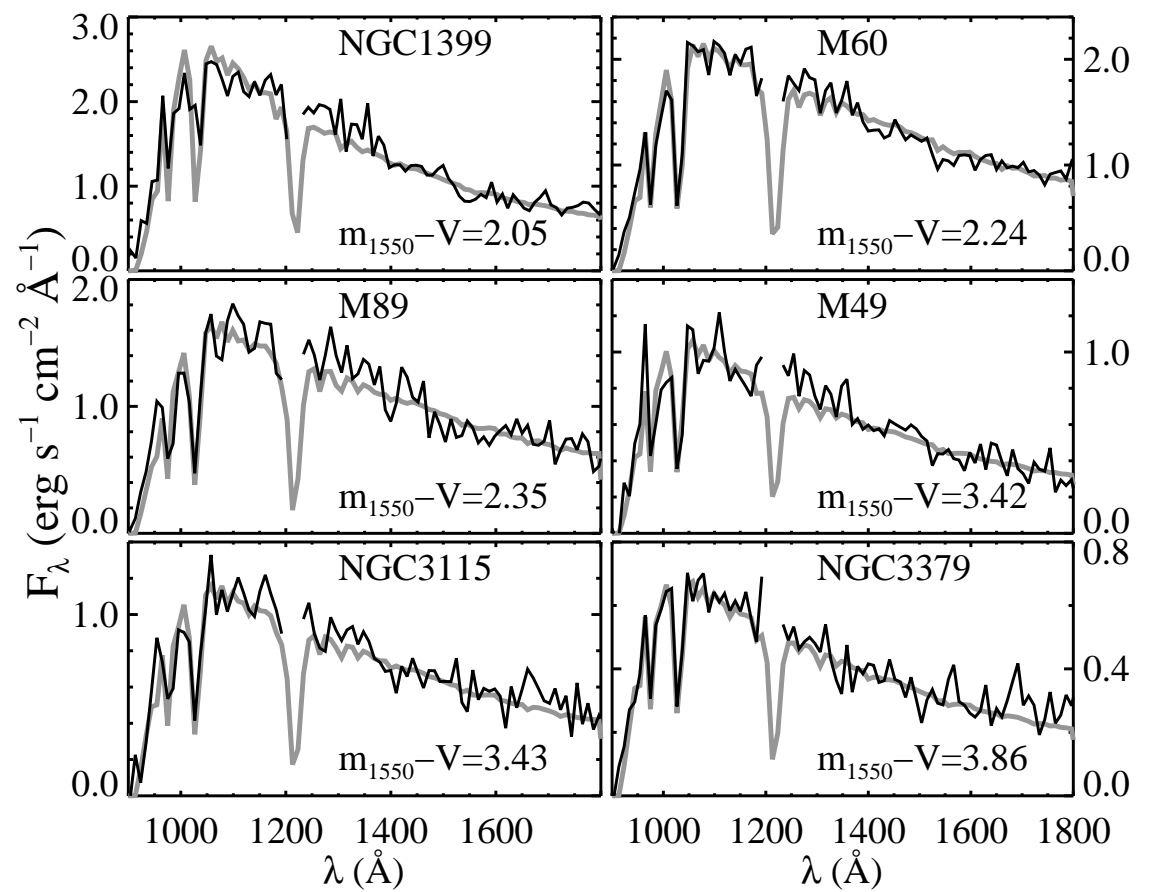

Figure 1. Spectra of six elliptical galaxies observed with the Hopkins Ultraviolet Telescope (black curves). Although the galaxies span a large range in $m_{1550}-V$ (labeled), they all appear very similar, and are well-matched by the integrated light (grey curves) of EHB stars and their progeny, spanning a narrow range of envelope mass.

abundance is $2 \%$ solar, the $\mathrm{Si}$ abundance is $13 \%$ solar, and the $\mathrm{N}$ abundance is $45 \%$ solar.

The UV upturn is positively correlated with optical metallicity indicators, contradicting the general tendency for the HB morphology to become redder at increasing metallicity. This has led to considerable debate about the metallicity of the hot stars responsible for the UV emission. In particular, Park \& Lee (1997) have argued that the UV upturn should be anticorrelated with the metallicity of the hot stars. In their view, the correlation between UV upturn and optical metallicity indicators is due to the more metal-rich galaxies being older and more massive. Others (e.g., Greggio \& Renzini, 1990; Bressan et al., 1994) have argued that the tendency for redder HB morphology at increasing metallicity is reversed at high metallicities, because of an associated increase in helium abundance and perhaps enhanced mass loss; in this case, the UV upturn should positively correlate with both optical and UV metallicity indicators. The metallicity of the EHB stars in NGC1399, the galaxy with the strongest known UV upturn, is clearly neither metal-rich nor metal-poor. 


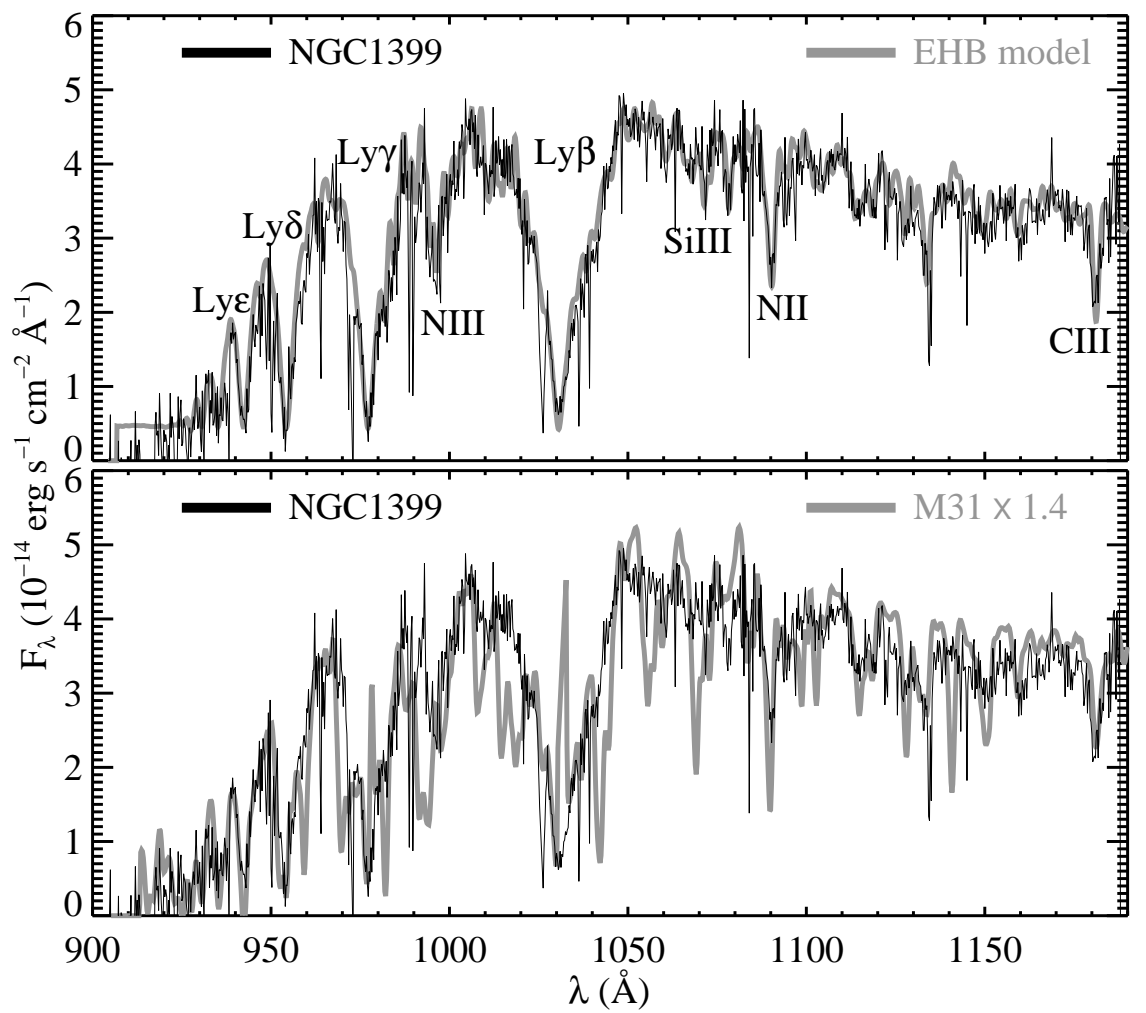

Figure 2. Top panel: The FUSE spectrum of NGC1399 (black curve) is compared to the best-fit EHB model (grey curve) from the earlier analysis of HUT data. Photospheric features are labeled; note the sharp Galactic interstellar features to the blue of several photospheric features. Bottom panel: The NGC1399 spectrum (black curve) is compared to the renormalized M31 spectrum (grey curve), convolved to the NGC1399 velocity dispersion. Although strong interstellar features complicate the analysis of the M31 spectrum, the CIII $\lambda 1175$ feature appears somewhat weaker in M31 than in NGC1399.

The surface abundance pattern of the EHB stars, derived from the UV spectra of elliptical galaxies, is probably affected by diffusion in the stellar atmospheres, as often found for sdB stars in the Galactic field. Nevertheless, it is still interesting to compare the metallicity in UV-strong galaxies with UV-weak galaxies, to look for general trends. Unfortunately, FUSE did not observe the UV-weak giant elliptical M49 before the onset of its hardware problems, and the spectrum of the nearby galaxy M32 is of very low signal-tonoise. Given the inability to point at Virgo, we replaced M49 in our program with the bulge of M31, which also has a fairly weak UV upturn. Its spectrum is shown in figure 2; because the bulge of M31 does not have the high velocity dispersion of NGC1399, the M31 spectrum has been convolved and renormalized appropriately for comparison. The spectrum of M31 is much more difficult to interpret than that of NGC1399; the redshift of NGC1399 sepa- 
rates the weak Galactic interstellar lines from the EHB photospheric lines, while the slight blueshift of M31 is not enough to separate these lines. Unlike NGC1399, which suffers from no foreground extinction, the foreground extinction toward the M31 nucleus is much stronger: $E(B-V)=0.08$ mag (Schlegel et al., 1998). Nevertheless, a few photospheric features have no interstellar counterpart, such as CIII $\lambda 1175$. This feature shows that the C abundance in M31 is also very weak - perhaps weaker than that in NGC1399. In general, the continuum shape is very similar in the two spectra. However, after dereddening, the M31 spectrum would appear somewhat hotter than the NGC1399 spectrum, suggesting a contribution from either hotter EHB stars and/or a higher contribution from post-AGB stars.

\section{UV Images of M32}

Because M32 has the weakest known UV upturn (Burstein et al., 1988), its IUE spectrum could have been explained entirely by relatively short-lived post-AGB stars instead of EHB stars. Early UV images of M32 and the M31 bulge, with the Faint Object Camera (FOC) on the Hubble Space Telescope (HST), resolved some of this UV emission into bright stars (Brown et al., 1998b), but later near-UV images with the Space Telescope Imaging Spectrograph (STIS) resolved the EHB stars in M32, demonstrating that they are responsible for nearly all of its UV emission (Brown et al., 2000b). Although no color information was available in those data, the near-UV luminosity function showed a sharp peak at the level of the EHB. Recently, we obtained far-UV observations of the same field with the HST/STIS, in order to construct a deep UV color-magnitude diagram (CMD) of M32 (figure 3). The CMD shows a well-populated EHB, confirming that the UV emission comes from a minority population of EHB stars $(\sim 2 \%$ of the total $\mathrm{HB})$. However, the images are missing many of the post-HB stars expected from canonical stellar evolution theory. Most of the HB stars in M32 lie on the red $\mathrm{HB}$, and those should produce hundreds of UV-bright post-AGB stars, yet the HST/STIS images have only a handful. Furthermore, there should be approximately 1-2 post-EHB stars (also known as AGB-Manqué stars) for every $10 \mathrm{EHB}$ stars, yet these post-EHB stars are also under-represented. This is shown by the simulation in figure 3 , which assumes a bimodal HB morphology that best reproduces the distribution of stars in the observed CMD. Although the number of EHB stars can be matched, no mass distribution reproduces the number of AGB-Manqué and post-AGB stars. Because the missing stars should be the brightest ones in the image, they cannot be missing due to instrumental effects or incompleteness. Instead, these post-HB stars likely evolve on much more rapid timescales than predicted by standard evolution theory. 


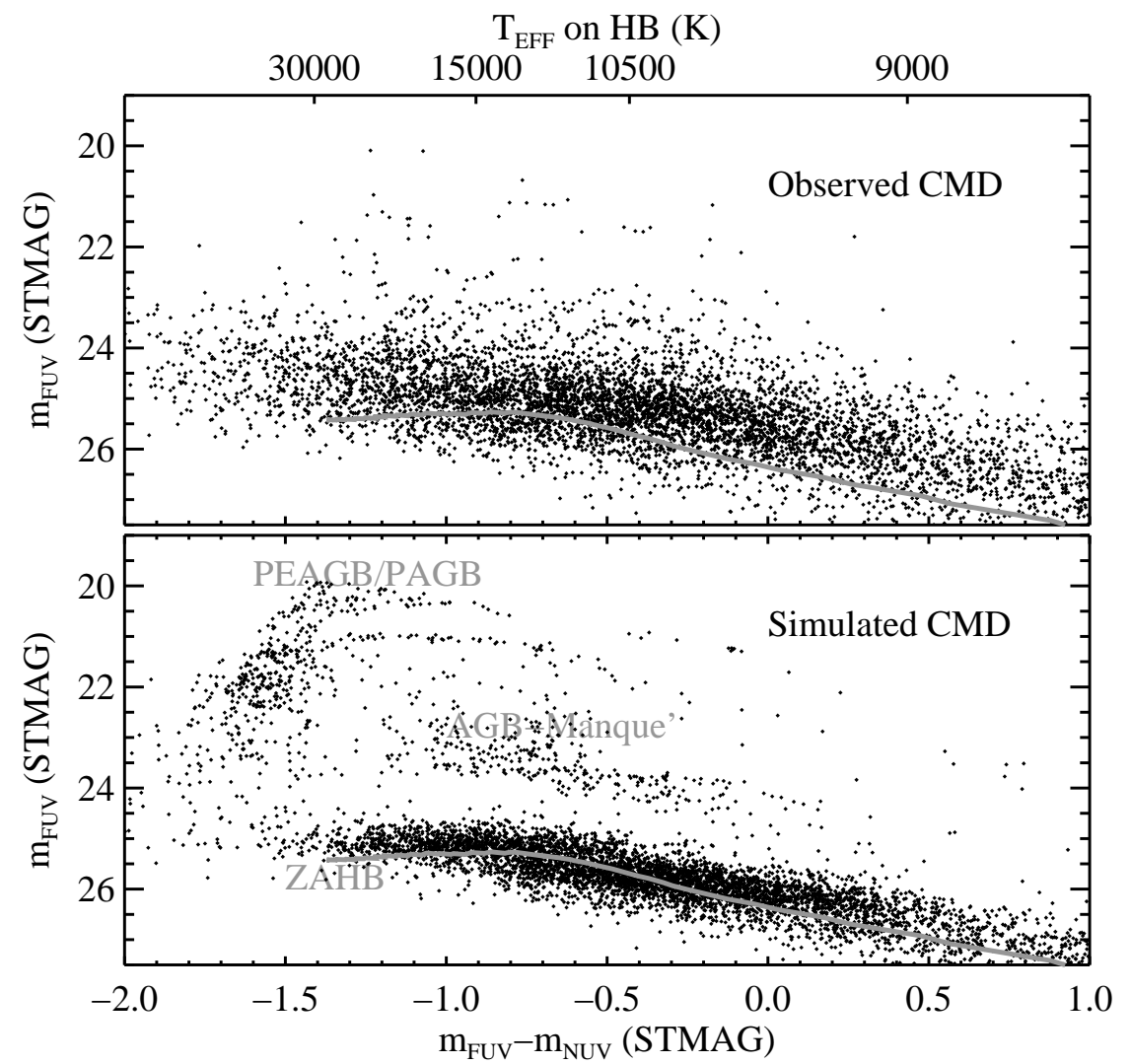

Figure 3. Top panel: The CMD constructed from the near-UV and far-UV images of M32, as observed by the HST/STIS (black points), compared to the predicted location of the zero-age HB (grey curve). Although the EHB is well-populated, the UV-bright stars above the EHB are under-represented. Bottom panel: The simulation that best reproduces the observed CMD, which assumes a bimodal HB morphology having a minority EHB population. Note the large numbers of UV-bright stars, and the clear gap between the EHB and AGB-Manqué stars, which are not seen in the observed CMD.

\section{The Evolution of the UV Upturn}

Because EHB stars are the source of the UV upturn, the UV upturn is expected to fade dramatically with increasing redshift as one looks to younger elliptical galaxies (Greggio \& Renzini, 1990; Tantalo et al., 1996). We have been undertaking a series of observations with HST to map the evolution of the UV upturn as a function of redshift, by observing galaxy clusters at $0.3<z<0.6$ (figure 4). Although early measurements were consistent with a relatively flat evolution out to $z \approx 0.4$ and a rapid fading at higher redshifts (Brown et al., 1998a; Brown et al., 2000a), recent observations (Brown et al., 2003) at $z=0.33$ show a UV upturn as weak as that at $z=0.55$. Because the earliest observations, at $z=0.375$, were the only ones that did not use 


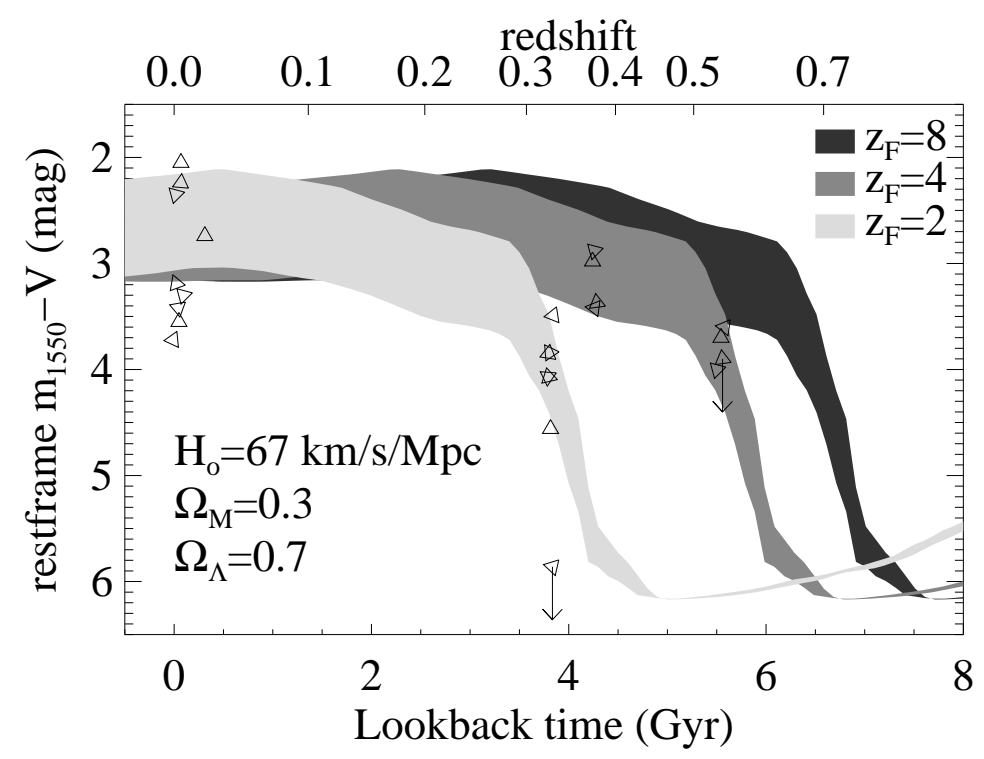

Figure 4. The evolution of the UV upturn as a function of redshift (triangles), with the expected evolution of this emission in giant elliptical galaxies (Tantalo et al., 1996), assuming three different formation redshifts $\left(z_{f}\right.$; labeled $)$.

a solar-blind camera, they might be systematically in error; setting aside the $z=0.375$ measurements, the remaining observations show a UV upturn that is weaker than that in the present epoch, but a relatively flat evolution with increasing lookback time. Taking the models at face value implies large variations in the formation epoch of giant elliptical galaxies in clusters, which is implausible (Brown et al., 2003). Although the onset of the UV upturn occurs at $\sim 6 \mathrm{Gyr}$ in these models, the formation of EHB stars is tied to a wide range of poorly constrained parameters (mass loss, metallicity, binary fraction, etc.), with the elliptical galaxies presenting the aggregate behavior at any one epoch. The "floor" in the UV emission seen at increasing redshift might indicate a wide dispersion in the parameters that govern EHB formation, or it might be that another source of UV emission is becoming dominant at increasing redshift as the EHB stars disappear (e.g., residual star formation). With our current understanding of EHB formation, the UV upturn remains a poorly calibrated age indicator. However, if galaxy ages are determined by independent methods, these surveys of the UV upturn could instead be used to constrain theories of EHB formation.

\section{Summary}

The past decade of observations in nearby galaxies has shown that EHB stars are the dominant source of the UV upturn, even in those galaxies with 
very weak UV emission, such as M32. We can now resolve EHB stars in galaxies beyond the Milky Way, out to the distance of M31 and its satellites. UV images of M32 show a well-populated EHB, but a surprising dearth of AGB-Manqué and post-AGB stars. As we look to elliptical galaxies at higher redshift, the UV upturn fades, but not as rapidly as might be expected, suggesting either a large dispersion in the parameters that govern the formation of EHB stars, or another source of UV emission that becomes dominant at earlier ages. Although the UV upturn may be the most sensitive indicator of age in an evolved population, it is a diagnostic that is poorly constrained by our current understanding of EHB stars.

\section{Acknowledgements}

The work presented herein was done in collaboration with A. Davidsen (JHU), H. Ferguson, R. Jedrzejewski, E. Smith (STScI), C. Bowers, B. Dorman, R. Kimble, R. Ohl, A. Sweigart (NASA/GSFC), R.M. Rich (UCLA), A. Renzini (ESO), J.-M. Deharveng (Laboratoire d'Astronomie Spatiale), R. O'Connell (U. of Virginia), and S.A. Stanford (LLNL). These observations were supported by NASA contract NAS 5-27000 to the Johns Hopkins University, NASA grant NAS 5-9696 to the Catholic University of America, NASA grant NAS 5-6499D to the Goddard Space Flight Center, and NASA grants NAG5-12278 and NAS 5-26555 to the Space Telescope Science Institute.

\section{References}

Bressan, A., Chiosi, C., \& Fagotto, F. 1994, ApJS, 94, 63

Brown, T.M., Bowers, C.W., Kimble, R.A., \& Ferguson, H.C. 2000a, ApJ, 529, L89.

Brown, T.M., Bowers, C.W., Kimble, R.A., Sweigart, A.V., \& Ferguson, H.C. 2000b, ApJ, 532, 308.

Brown, T.M., Ferguson, H.C., \& Davidsen, A.F. 1995, ApJ, 454, L15.

Brown, T.M., Ferguson, H.C., Davidsen, A.F. \& Dorman, B. 1997, ApJ, 482, 685.

Brown, T.M., Ferguson, H.C., Deharveng, J.-M., \& Jedrzejewski, R.I. 1998a, ApJ, 508, L139.

Brown, T.M., Ferguson, H.C., O’Connell, R.W., \& Ohl, R.G. 2002, ApJ, 568, L19.

Brown, T.M., Ferguson, H.C., Smith, E., Bowers, C.W., Kimble, R.A., Renzini, A., \& Rich, R.M. 2003, ApJ, 584, L69.

Brown, T.M., Ferguson, H.C., Stanford, S.A., \& Deharveng, J.-M. 1998b, ApJ, 504, 113.

Burstein, D., Bertola, F., Buson, L.M., Faber, S.M., \& Lauer, T.R. 1988, ApJ, 328, 440.

Code, A.D. 1969, PASP, 81, 475.

Ferguson, H.C., et al. 1991, ApJ, 382, 69.

Greggio, L., \& Renzini, A. 1990,ApJ, 364, 35

Park, J.-H., \& Lee, Y.-W. 1997, ApJ, 476, 28.

Schlegel, D.J., Finkbeiner, D.P., \& Davis, M. 1998, ApJ, 500, 525.

Tantalo, R., Chiosi, C., Bressan, A., \& Fagotto, F. 1996, A\&A, 311, 361. 